\title{
Galactic globular cluster stars: From theory to observation
}

\author{
S. Cassisi ${ }^{1,2,3}$, V. Castellani ${ }^{4}$, S. Degl'Innocenti ${ }^{4,5}$, M. Salaris ${ }^{6}$, and A. Weiss ${ }^{6}$ \\ 1 Osservatorio Astronomico di Collurania, via Mentore Maggini, I-64100 Teramo, Italy \\ 2 Dipartimento di Fisica, Universitá de L'Aquila, via Vetoio, I-67010 L'Aquila, Italy \\ 3 Osservatorio Astronomico di Capodimonte, via Moiariello, I-80131 Napoli, Italy \\ 4 Dipartimento di Fisica, Universitá di Pisa, piazza Torricelli 2, I-56126 Pisa, Italy \\ 5 Istituto Nazionale di Fisica Nucleare, Sezione di Ferrara, via Paradiso 12, I-44100 Ferrara, Italy \\ ${ }^{6}$ Max Plank Institut for Astrophysics, Karl Schwarzschild strasse 1, D-85470 Garching b. Munchen, Germany
}

Received March 23; accepted July 23, 1998

\begin{abstract}
We use evolutionary calculations presented in a recent paper (Cassisi et al. 1997a: hereinafter Paper I) to predict $B, V, I$ magnitudes for stars in galactic globulars. The effect of the adopted mixing length on stellar magnitudes and colors is discussed, showing that the uncertainty on such a theoretical parameter prevents the use of MS stars as bona fide theoretical standard candles. However, comparison with Hipparcos data for field subdwarfs discloses a substantial agreement between theory and observation. Present predictions concerning the magnitude of TO and of HB stars are compared with similar results appeared in the recent literature. We find that our predictions about the dependence on metallicity of ZAHB magnitudes appear in good agreement with observational constraints as recently discussed by Gratton et al. (1997c). We present and discuss a theoretical calibration of the difference in magnitude between $\mathrm{HB}$ and TO as evaluated with or without element sedimentation. The effect of a variation of the original helium content on the magnitude of MS, TO and HB stars is explored and discussed. Finally we use theoretical HB magnitudes to best fit the CM diagram of M 68 and M 5, taken as representative of metal poor and intermediate metallicity galactic globulars, deriving an age of $11 \pm 1.0 \mathrm{Gyr}$ and $10 \pm 1.0 \mathrm{Gyr}$, respectively, for the adopted chemical compositions, plus an additional uncertainty of \pm 1.4 Gyr if the uncertainty on the chemical composition is taken into account. This result is discussed on the basis of current evaluations concerning cluster ages and distance moduli.
\end{abstract}

Key words: stars: evolution - HR diagram - horizontalbranch — Population II

Send offprint requests to: S. Degl'Innocenti (Dipartimento di Fisica, Università di Pisa) scilla@astr18pi.difi.unipi.it

\section{Introduction}

Following the effort undertaken by Hubble Space Telescope for improving the determination of the Hubble constant and by taking advantage of several improvements of the input physics a number of authors have recently revisited the prediction of stellar evolutionary theory to re-evaluate the age of galactic globular clusters (see, e.g., VandenBerg et al. 1996; D'Antona et al. 1997; Salaris et al. 1997; Brocato et al. 1997; Chaboyer et al. 1998; Salaris \& Weiss 1998a,b). In a previous paper (Cassisi et al. 1998: hereinafter Paper I) we discussed on purely theoretical grounds the effect of the improved physical inputs on stellar evolutionary models, evaluating the additional influence of element diffusion on the evolutionary history of such stars. In this paper we take advantage of such an homogeneous set of stellar models to present theoretical predictions concerning observational magnitudes and colors, and to discuss the theoretical scenario leading to the evaluation of cluster ages.

Such age evaluations, as primarily based on the calibration of the Turn-Off luminosity in terms of the cluster age, requires the adoption of suitable standard candles belonging to the cluster, as given either by MS or by $\mathrm{HB}$ stars. In the last case one is dealing with the so called $\Delta V$ method, which is only formally independent of the cluster distance modulus. In all cases, connecting theory to ages requires several steps worth being discussed in some details. Thus, presenting the evolutionary results, we will also investigate the degree of reliability concerning theoretical predictions about the magnitude of MS, TO and HB stars. The main aim of this investigation is to point out theoretical uncertainties but also to show how reliable the new models are, testing the results with available observational constraints and, in particular, with the absolute magnitudes of metal poor stars recently provided by Hipparcos. 


\section{From theory to observation}

In order to cover with sufficient details the range in metallicity $Z=210^{-4}-610^{-3}$ which appears adequate for galactic globulars, computations presented in Paper I have been supplemented with an additional set of stellar models with $Z=0.0006$. As in Paper I, evolutionary models have been computed by adopting an amount of original $\mathrm{He}$ $Y=0.23$ and with a mixing length parameter $l=1.6 \mathrm{Hp}$. Data for these new models are reported in the Appendix of this paper.

On the basis of these new results, in this paper we will rely on a grid of evolutionary models covering, at least, the range of cluster ages 8-18 Gyr for the assumed metallicities $Z=0.0002,0.0006,0.001,0.006$. The computations have been performed both by including or neglecting element sedimentation. In addition, for $Z=0.001$ we computed two set of evolutionary tracks with element sedimentation, under the same assumption about the mixing length but with $Y=0.21$ or 0.25 , respectively. These models will allow to discuss the influence of original $\mathrm{He}$ on models where element diffusion is taken into account. According to the usual procedure, the evolutionary models have been used to derive cluster isochrones in the $\log L$, $\log T_{\text {e }}$ plane.

To allow a comparison with observation, this theoretical scenario has to be implemented with suitable procedures transforming the theoretical quantities $L$ (luminosity) and $T_{\mathrm{e}}$ (effective temperature) into reliable predictions about magnitudes in selected photometric bands. To look into this problem Fig. 1 shows our $Z=0.000212 \mathrm{Gyr}$ isochrone (with diffusion) as transformed into the CM diagram according to selected available choices about the color-temperature and magnitude luminosity transformations. One finds that the most recent evaluations given by Castelli et al. (1997a,b) are very similar to previous evaluations given by Buser \& Kurucz $(1978,1992)$ all along the TO region as well as in the redder portion of the HB, whereas Kurucz (1992) gives slightly redder TO colors. In all cases one finds that the difference affects mainly the colors, whereas bolometric corrections appear remarkably similar. Thus $V$ magnitudes appears independent of the choice of the models.

If not otherwise advised, in this paper we will rely on the model atmospheres by Castelli et al. (1997a,b). Here we note that preliminary computations (Castelli, private communication) for the case $Z=0.0002$ have already shown that the model atmospheres can be further improved. As a matter of fact, one finds that when both the new solar abundances and the enhancement of $\alpha$-elements is taken into account, model atmospheres for metal poor stars $([\mathrm{Fe} / \mathrm{H}]=-2.0)$ reach a better agreement with available empirical estimate by Alonso et al. (1996). Fortunately, the same Fig. 1 shows that such improvement affects only the slope of the MS at the lower luminosity,

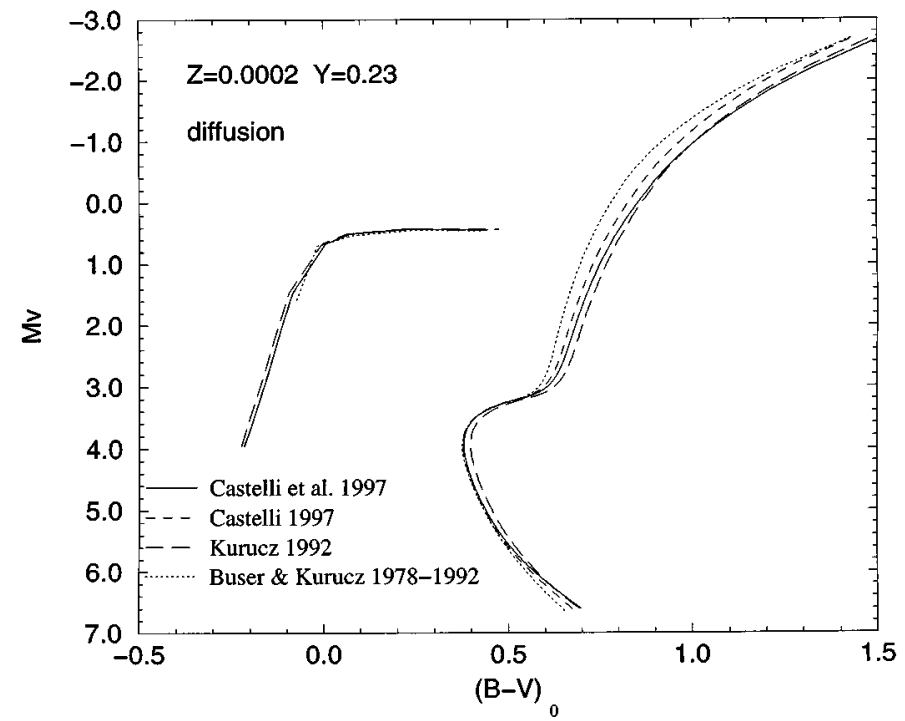

Fig. 1. The run of the theoretical $12 \mathrm{Gyr}$ isochrone in the $V$, $B-V$ diagram for the labeled choices about the adopted model atmospheres

leaving in particular unaffected the predicted magnitude of candidate candle stars.

As is well known, MS models are also affected by an intrinsic uncertainty due to the assumptions about the value of the mixing length parameter. The effect of varying the adopted mixing length within the (reasonable) interval $l=1.3-2.0 \mathrm{Hp}$ is shown in Fig. 2, where we report selected isochrones for the labeled values of ages and mixing length parameters. One finds that for ages ranging from 9 to 15 Gyr the MS color at $M_{v}=6.0$ decreases by about $\Delta(B-V) \sim 0.02 \mathrm{mag}$, whereas decreasing the mixing length from 2.0 down to $1.3 \mathrm{Hp}$ (the local pressure scale height) gives again a decrease of this color of about $\Delta(B-V) \sim 0.03 \mathrm{mag}$, depending on the cluster age. Thus the color of the MS at $M_{v}=6.0$ is far from being a firm prediction of theory. The same figure shows that the magnitude of the MS at $B-V=0.55$ (i.e. around theoretical predictions for $M_{v}=6$ ) for a given cluster metallicity can move by about $0.28 \mathrm{mag}(\sim 0.18 \mathrm{mag}$ for a variation from 1.3 to $2.0 \mathrm{Hp}$ and $\sim 0.1 \mathrm{mag}$ for a difference in age from 9 to 15 Gyr), preventing the use of theoretical MS models as precise distance calibrators.

When moving toward more advanced evolutionary phases, as already noticed by Chaboyer (1995), the calibration of TO magnitudes in terms of cluster ages does depend on the assumptions about the mixing length parameter. One finds that such a dependence increases when the cluster ages and/or the metallicity is increased, for the simple reason that in both cases TO stars become cooler and, thus, more affected by external convection. As a result, the most metal poor and younger TO (i.e., the hottest TO) are barely affected by the treatment of convection all over the explored range of mixing length parameters $(1.3 \div 2.0 \mathrm{Hp})$. As an example, for $Z=0.0002$ 
and for the age $t=9 \mathrm{Gyr}$, a change from $l=1.3$ to 2.0 $\mathrm{Hp}$ moves the TO magnitude by only $0.02 \mathrm{mag}$. However, for the same assumed metallicity, at $t=14 \mathrm{Gyr}$, the same variation of the mixing length parameter gives a difference in the TO magnitude of the order of $0.1 \mathrm{mag}$.

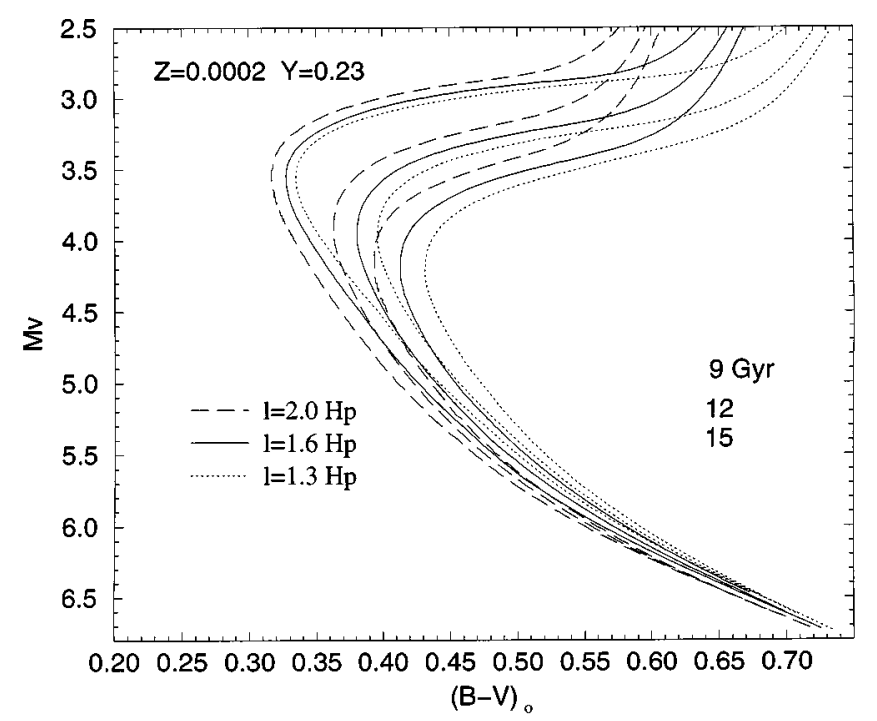

Fig. 2. The 9, 12 and 15 Gyr isochrones as computed under the labeled assumptions about the mixing length parameter and translated in the CM diagram according to Castelli et al. $(1997 \mathrm{a}, \mathrm{b})$

According to the quoted evidences, we will proceed in the discussion of theoretical predictions with the warning that discussed magnitudes should be reliable, whereas colors are affected by the discussed uncertainties in both stellar and atmospheric models. Detailed data for the computed isochrones are available by anonymous ftp at astr18pi.difi.unipi.it (/pub/globular).

In the next section we discuss low mass stars during the major phase of $\mathrm{H}$ burning, namely from the initial main sequence to the subgiant phase, just after the exhaustion of central $\mathrm{H}$. We selected this evolutionary phase according to the evidence that the corresponding stellar models rely on the "minimum" input physics as given by an equation of state (EOS) not too far from a perfect gas, by radiative opacities and, finally, by sufficiently well known $\mathrm{H}$ burning rates (see, e.g., Brocato et al. 1998) Correspondingly, the quoted evolutionary phases have to be regarded as the most solid predictions of theory. Section 4 will deal with a discussion of more advanced evolutionary phases, from the red giant branch to the AGB through the phase of central He burning, where more physics has to be added, like neutrino production by weak interactions or the physical behavior of electron-degenerate matter. In both cases we find that theoretical predictions reach a good agreement with observational constraints. Following such an agreement, we discuss relevant evolutionary features and, in particular, the estimate of cluster ages based on the HB luminosity level. A brief final discussion closes the paper.

\section{3. $\mathrm{H}$ burning phases}

As a first test of theoretical results, Figs. 3a to c shows a comparison between isochrones (with sedimentation) and Hipparcos data (Gratton et al. 1997; Chaboyer et al. 1998) for field subdwarfs in selected intervals of $[\mathrm{Fe} / \mathrm{H}]$ values. Each panel compares isochrones at $t=10$ and 12 Gyr and for a given $[\mathrm{Fe} / \mathrm{H}]$ value with subdwarfs with $[\mathrm{Fe} / \mathrm{H}]$ estimates within \pm 0.1 dex. Triangles in each panel show the estimated shift in the isochrone if the suggested enhancement in $\alpha$-elements $[\alpha / \mathrm{Fe}] \simeq 0.3$ (Carretta \& Gratton 1997; Gratton et al. 1998a) is accounted as an increase in the total value of $Z$. For the sake of comparison, each panel shows also the location of the MS loci but for different metallicities.

Inspection of the three panels shows that $\alpha$-elements play a minor role, since the corresponding shift of the isochrone lies within the observational errors. Bearing in mind that sedimentation does not affect the location of MS stars, one finds that the agreement between theory and observation appears as fine as it can be expected according to the standard errors in absolute magnitudes and the spread in metallicity. One finds that the popular Reid's statement "current models overestimate the change in luminosity with decreasing abundance for extreme metal poor subdwarf" is hardly supported. In fact, the three panels of Fig. 3 suggest that no contradiction appears between extant theory and Hipparcos observations.

Figure 4 shows the comparison between present predictions of $B-V$ colors at $M_{v}=6.0$ and Hipparcos observational data as presented by Gratton et al. (1997). One finds that theoretical data appear in satisfactory agreement with observations, whose error appears of the same order of magnitude of the theoretical uncertainty discussed in the previous section. The best fit to the theoretical data gives:

$(B-V)=0.890+0.256[\mathrm{Fe} / \mathrm{H}]+0.048[\mathrm{Fe} / \mathrm{H}]^{2}$

(at $\left.M_{v}=6.0\right)$

which practically overlaps the relation given by Gratton et al. (1997a) except for a difference in the zero point by $\Delta(B-V)=0.014$. As an alternative approach to the effect of metallicity one finds for the MS magnitude at $(B-V)=0.6$ :

$M_{v}=3.889-2.160[\mathrm{Fe} / \mathrm{H}]-0.509[\mathrm{Fe} / \mathrm{H}]^{2}$

$($ at $(B-V)=0.6)$.

One can use either of these two relations for correcting the metallicity effects in data in Fig. 3, by shifting the observational data to the $[\mathrm{Fe} / \mathrm{H}]$ value of the computed isochrone. However, one finds that such a procedure does not improve the fitting given in Fig. 3, as an evidence that parallax errors (i.e., errors in absolute magnitudes) overcome the effect of metallicity in the chosen dwarf samples. 


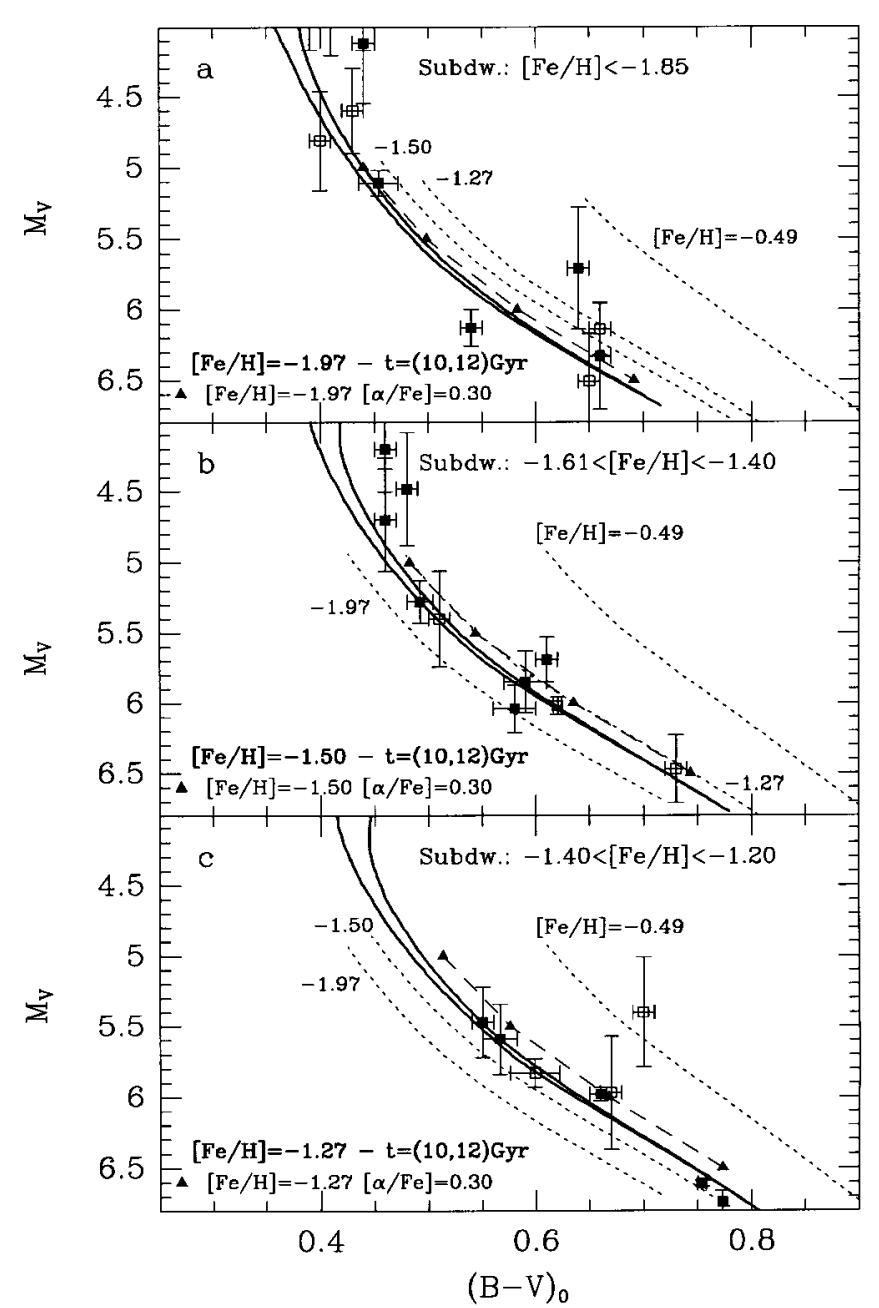

Fig. 3. Comparison of present isochrones with Hipparcos subdwarf absolute magnitudes (Gratton et al. 1997). Each panel shows the run of the 10 and 12 Gyr isochrones (full lines) for the given value of $[\mathrm{Fe} / \mathrm{H}]$. Triangles (and the dashed line) show the shift of the 10 Gyr isochrones caused by an enhancement $[\alpha / \mathrm{Fe}] \simeq 0.3$. As a comparison, dotted lines show the run of $10 \mathrm{Gyr}$ isochrones with different $[\mathrm{Fe} / \mathrm{H}]$, as labeled. Filled squares and open squares indicate single stars and detected or suspected binary stars, respectively

When moving from the MS to more advanced phases, Fig. 5 gives TO $V$-magnitudes as a function of age for the various adopted metallicities. One finds that for each given metallicity the TO magnitudes can be arranged as a linear function of the logarithm of the age, with a maximum error (for $Z=0.001$ ) not exceeding 0.4 Gyr. Table 1 gives the coefficients of the linear relations connecting the TO visual magnitudes with cluster ages (in Gyr) for isochrones with element diffusion taken into account. Figure 6 shows the comparison between current predictions for the TO magnitudes at $t=10 \mathrm{Gyr}$ and for the four adopted metallicities with similar results in the literature. As already predicted in Paper I, one finds that present evaluations tend to decrease the cluster age for any given TO mag-

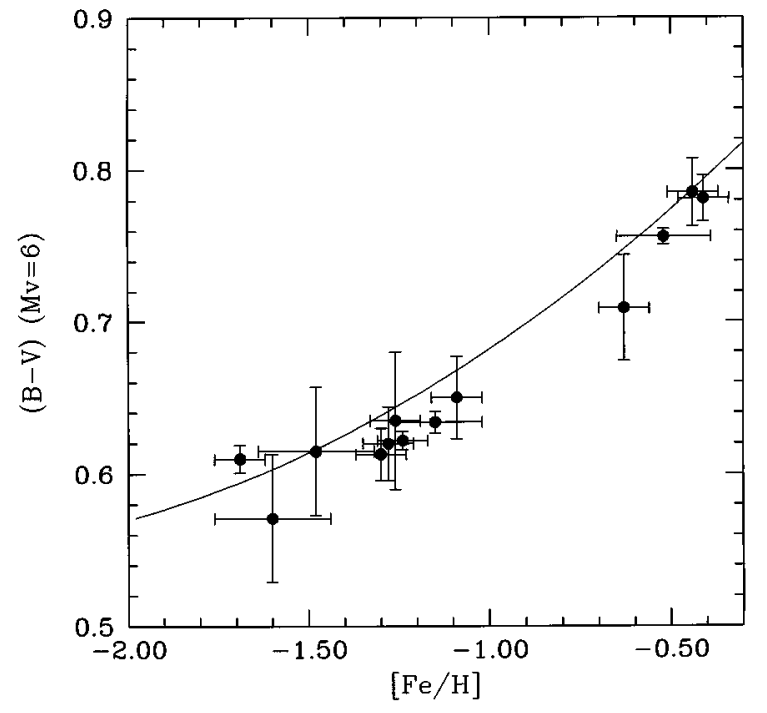

Fig. 4. Present predictions of $B-V$ colors at $M_{v}=6.0$ for the various metallicities compared with observational data given by Gratton et al. (1997a) on the basis of Hipparcos observations

Table 1. Coefficients of the linear relation: $M_{v}^{\text {TO }}=a \log ($ age $)$ $+b$ which connects the visual magnitude at the isochrone TO $\left(M_{v}^{\mathrm{TO}}\right)$ with the TO age for present models with element diffusion and $Y=0.23$. Age is in Gyr, metallicity as indicated

\begin{tabular}{lcc}
\hline \hline & & \\
$Z$ & $a$ & $b$ \\
\hline 0.0002 & 2.70 & 0.98 \\
0.0006 & 2.55 & 1.30 \\
0.001 & 2.48 & 1.46 \\
0.006 & 2.33 & 1.83 \\
\hline \hline
\end{tabular}

nitude, the differences in Fig. 6 being mainly the consequence of the difference already discussed in Paper I concerning theoretical luminosities. In passing we note that at the larger metallicities present magnitudes do not overlap Mazzitelli et al. (1995) predictions, as occurred for luminosities. This is mainly due to a small difference in adopted visual magnitude of the Sun: MDC adopted $M_{v \odot}=$ 4.79 mag against our preferred canonical value $M_{v \odot}=$ 4.82 mag.

As already known, the TO magnitude, which is defined as the magnitude of the bluest point of the isochrone, is a difficult observational parameter in particular in the most metal poor clusters. Moreover, small variations in the shape of the theoretical isochrone can produce relevant variation in the magnitude of the nominal TO. This explains the small departures from linearity disclosed by data plotted in Fig. 5. In a recent paper Chaboyer et al. (1996) suggested a different calibrator of the globular cluster age, as given by the visual magnitude of the so called BTO point, that is the point brighter than the TO and redder by $\Delta(B-V)=0.05$. The main advantage of 


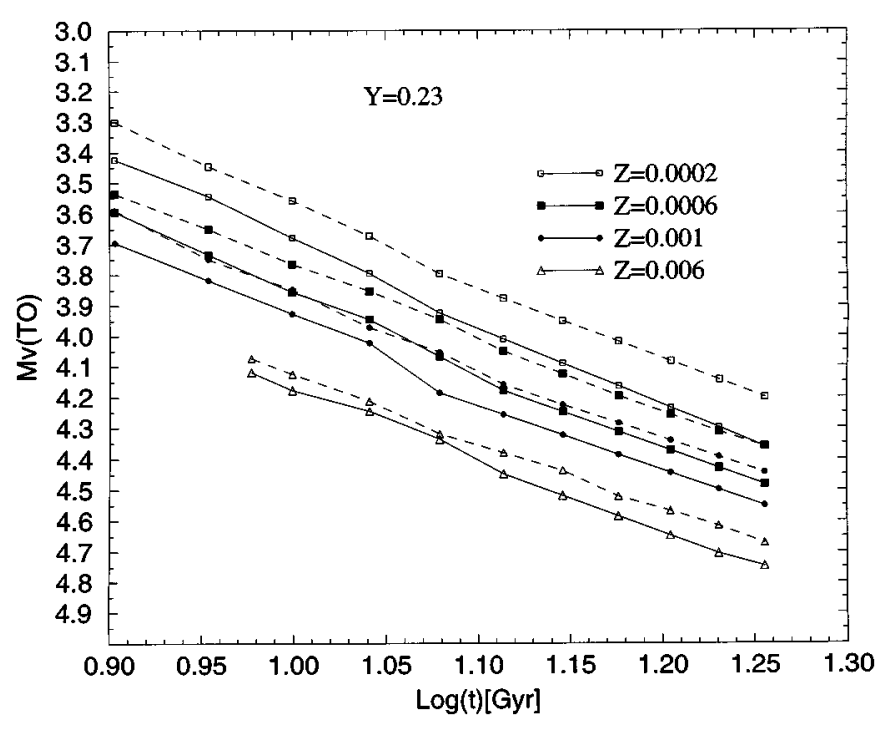

Fig. 5. Visual magnitude of the TO isochrones as a function of the logarithm of the age, for the labeled metallicities for models with (solid line) and without (dashed line) element diffusion

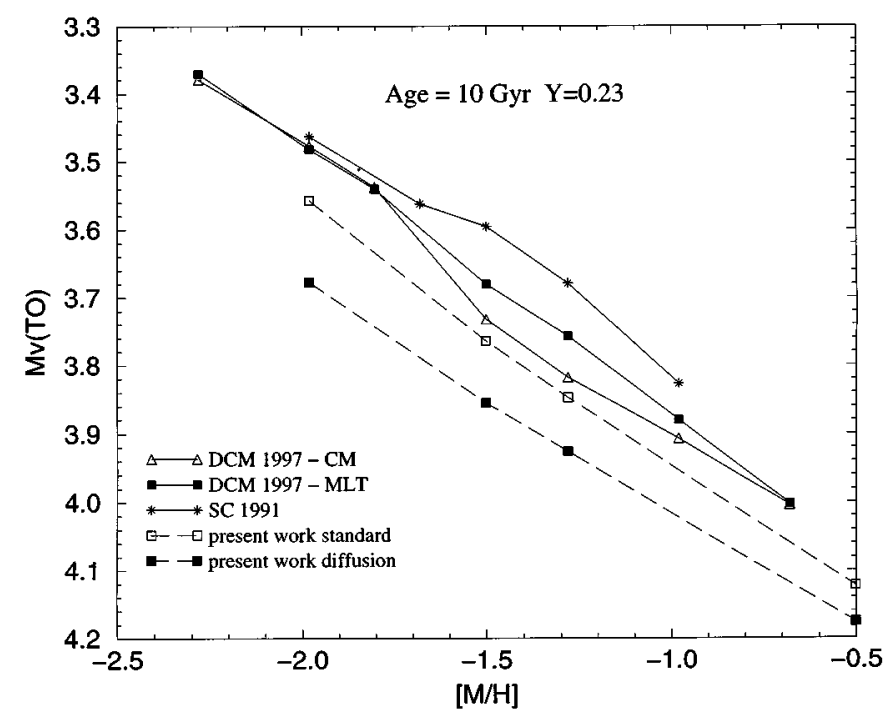

Fig. 6. TO visual magnitudes for $t=10 \mathrm{Gyr}$ as a function of the metallicity for present models as compared with similar results available in the current literature. For DCM 1997 models CM indicates the adoption by the authors of the Canuto \& Mazzitelli (1991) treatment of overadiabatic convection while MLT indicates the adoption of the usual mixing length theory

$M_{v}$ (BTO) is that this point is not in the vertical turn-off region and thus it is easier found in the observed color magnitude diagram. Figure 7 shows that the visual magnitude of theoretical BTO appears much more regularly depending on the cluster age than $M_{v}(\mathrm{TO})$ does, further supporting the use of such a parameter.

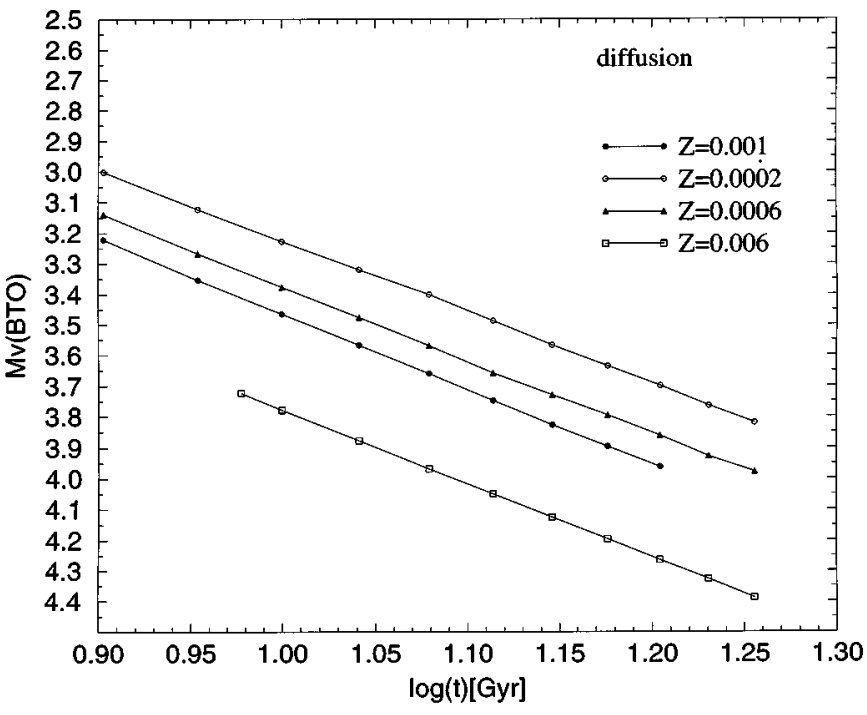

Fig. 7. Visual magnitude at the isochrone BTO for present models with diffusion as a function of the logarithm of the age, for the labeled metallicities

\section{Horizontal branches}

As a preliminary point, let us recall that in Paper I HB models have been computed assuming initial He core masses from a $0.8 M_{\odot}$ progenitor. Strictly speaking, according to data given in the same paper, this implies an age of the order of $11 \mathrm{Gyr}$ for the most metal poor cluster, increasing up to 16 Gyr for $Z=0.006$. In Paper I we already discussed this issue, reaching the conclusion that the expected variation of HB luminosity can be neglected over a reasonable range of ages. Now we add that detailed numerical experiments show that a variation of the cluster ages of \pm 5 Gyr gives a variation in the predicted HB magnitude of $\pm 0.02 \mathrm{mag}$. According to the calibration given in Fig. 5 a similar error in the cluster distance modulus would produce an error in the cluster age not larger than 0.3 Gyr which, however, could be easily taken into account whenever a better precision would be required.

Table 2 gives details for HB structures in the observational plane at the various metallicities. Selected evolutionary quantities for the new HB models with $Z=0.0006$ are given in the Appendix of this paper. A comparison of theoretical predictions for $\mathrm{HB}$ with Hipparcos data has been already presented in Paper I. Figure 8 compares the magnitude of the ZAHB (at $\log T_{\mathrm{e}}=3.85$ ) with previous evaluations on the matter. One recognizes the not negligible increase in the predicted HB luminosity induced by the improved physics as well as the fair agreement, toward the lower metallicities, with the recent computations by Caloi et al. (1997). As already predicted (Castellani et al. 1991) one finds that the dependence of the ZAHB luminosity on the metallicity increases with metallicity. The best fit of data in Fig. 8 gives: 
Table 2. Selected evolutionary quantities in the observational plane for ZAHB models with $Y=0.23$ and element diffusion. Masses are in solar units

\begin{tabular}{|c|c|c|c|c|c|c|c|c|c|c|c|c|c|c|c|c|}
\hline \multicolumn{17}{|c|}{$\begin{array}{c}Z=0.0002 Y_{\mathrm{HB}}=0.226 \\
M_{\mathrm{c}}=0.515 M_{\odot}\end{array}$} \\
\hline$M$ & 0.530 & 0.540 & 0.550 & 0.560 & 0.580 & 0.600 & 0.620 & & .650 & 0.670 & 0.690 & 0.700 & 0.720 & 0.750 & 0.770 & 0.800 \\
\hline$M_{V}$ & 3.954 & 3.658 & 3.415 & - & 2.806 & 2.436 & 2.045 & & 1.457 & - & - & 0.663 & 0.499 & 0.423 & 0.427 & 70.439 \\
\hline$B-V$ & -0.214 & -0.196 & -0.183 & - & -0.150 & -0.132 & -0.11 & & 0.081 & - & - & 0.008 & 0.076 & 0.233 & 0.327 & 70.446 \\
\hline \multicolumn{17}{|c|}{$\begin{array}{c}Z=0.0006 Y_{\mathrm{HB}}=0.229 \\
M_{\mathrm{c}}=0.509 M_{\odot}\end{array}$} \\
\hline$M$ & 0.530 & 0.540 & 0.550 & 0.560 & 0.580 & 0.600 & 0.620 & & .650 & 0.670 & 0.690 & 0.700 & 0.720 & 0.750 & 0.770 & 0.800 \\
\hline$M_{V}$ & 3.762 & - & 3.084 & - & - & 1.948 & - & & 0.650 & 0.525 & 0.516 & - & - & 0.523 & - & 0.487 \\
\hline$B-V$ & -0.202 & - & -0.162 & - & - & -0.108 & - & & .027 & 0.139 & 0.331 & - & - & 0.612 & - & 0.660 \\
\hline \multicolumn{17}{|c|}{$\begin{array}{c}Z=0.001 Y_{\mathrm{HB}}=0.229 \\
M_{\mathrm{c}}=0.507 M_{\odot}\end{array}$} \\
\hline M & 0.530 & 0.540 & 0.550 & 0.560 & 0.580 & 0.600 & 0.620 & & .650 & 0.670 & 0.690 & 0.700 & 0.720 & 0.750 & 0.770 & 0.800 \\
\hline$M_{V}$ & 3.673 & - & 3.099 & - & - & 1.702 & - & & .548 & - & - & 0.590 & - & 0.555 & - & - \\
\hline$B-V$ & -0.202 & - & -0.171 & - & - & -0.102 & - & & .187 & - & - & 0.643 & - & 0.718 & - & - \\
\hline \multicolumn{17}{|c|}{$\begin{array}{c}Z=0.006 Y_{\mathrm{HB}}=0.231 \\
M_{\mathrm{c}}=0.500 M_{\odot} \\
\end{array}$} \\
\hline M & 0.530 & 0.540 & 0.550 & 0.560 & 0.580 & 0.600 & 0.620 & & .650 & 0.670 & 0.690 & 0.700 & 0.720 & 0.750 & 0.770 & 0.800 \\
\hline$M_{V}$ & 3.101 & 2.748 & 2.368 & 1.904 & 0.793 & 0.833 & - & & - & - & - & 0.781 & - & - & - & 0.747 \\
\hline$B-V$ & -0.157 & -0.138 & -0.118 & -0.094 & 0.360 & 0.610 & - & & - & - & - & 0.898 & - & - & - & 0.942 \\
\hline
\end{tabular}

$M_{v}=0.993+0.461[\mathrm{Fe} / \mathrm{H}]+0.087[\mathrm{Fe} / \mathrm{H}]^{2}$

which reproduces the theoretical predictions for ZAHB with diffusion within less than $0.01 \mathrm{mag}$ all over the assumed range of metallicities. If diffusion is not taken into account the above magnitude has to be decreased by about $0.04 \mathrm{mag}$.

For metallicities lower than $Z=0.001$ the above relation can be approximated by a linear relation, as usually adopted in the literature. We find:
$M_{v}=0.18[\mathrm{Fe} / \mathrm{H}]+0.74$
$M_{v}=0.18[\mathrm{Fe} / \mathrm{H}]+0.77$
(no diffusion)
(diffusion).

These results can be usefully compared with the fairly large amount of observational relations presented in the literature:

$$
\begin{aligned}
& M_{v}=0.15[\mathrm{Fe} / \mathrm{H}]+1.01 \\
& M_{v}=0.15[\mathrm{Fe} / \mathrm{H}]+0.73 \\
& M_{v}=0.15[\mathrm{Fe} / \mathrm{H}]+0.84 \\
& M_{v}=0.18[\mathrm{Fe} / \mathrm{H}]+0.74 \\
& M_{v}=0.19[\mathrm{Fe} / \mathrm{H}]+0.97 \\
& M_{v}=0.23[\mathrm{Fe} / \mathrm{H}]+0.83 \\
& M_{v}=0.30[\mathrm{Fe} / \mathrm{H}]+0.94
\end{aligned}
$$

(Carney et al. 1992)

(Walker 1992)

(De Santis 1996)

(Gratton et al. 1998b)

(Clementini et al. 1995)

(Chaboyer et al. 1998)

(Sandage 1993).

It appears that present predictions show a dependence on the metallicity in reasonable agreement with the evaluation by Walker (1992) and the more recent evaluation given by Gratton et al. (1998b) based on Hipparcos re-

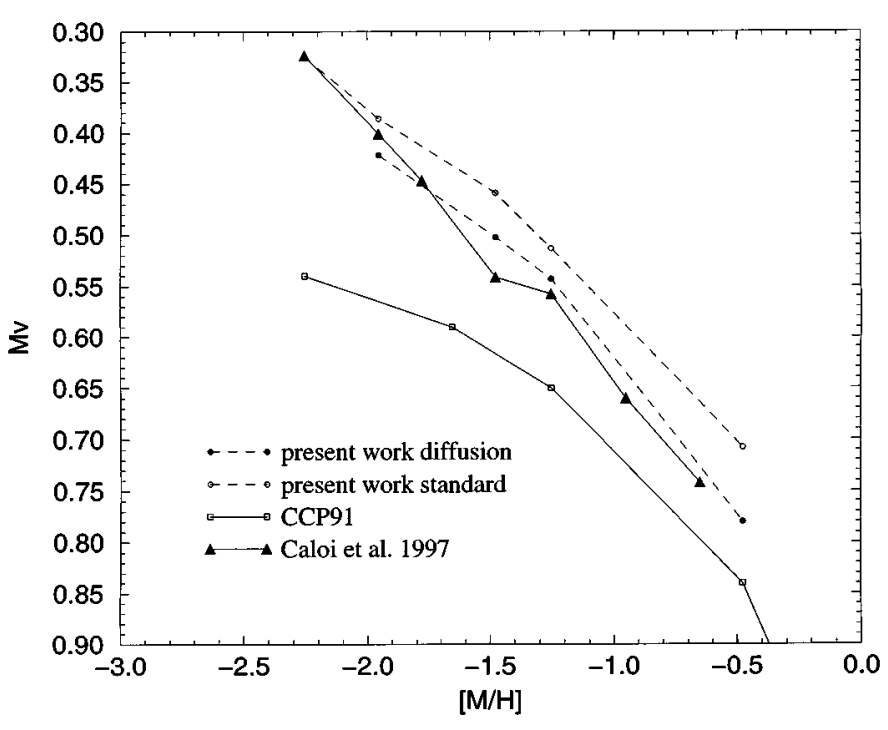

Fig. 8. Visual magnitude of the ZAHB at $\log T_{\mathrm{e}}=3.85$ as a function of the metallicity for present models with (solid line) and without diffusion (dashed line) compared with values for the same quantity available in the literature. CCP91 indicates models by Castellani et al. (1991)

sults, and definitely smaller than required by Sandage in his scenario for explaining the Oosterhoff dichotomy. However, before comparing the zero point of the magnitudes one has to recall that our previous relations refer to the ZAHB luminosity level, whereas observational data 
refer to the mean luminosity of the $\mathrm{HB}$ at the color of the RR Lyrae Gap. The connection between the two luminosity level has been already discussed in several paper (see Caputo et al. 1987; Carney et al. 1992; Cassisi \& Salaris 1997). One can safely assume $\Delta M_{v}=0.08 \mathrm{mag}$ as a suitable estimate of the difference in magnitude between $\mathrm{RR}$ and ZAHB. Thus our zero points will become $M_{v}=0.69$ (diffusion) or $M_{v}=0.66$ (no diffusion), respectively. One finds that we are predicting $\mathrm{HB}$ with the same dependence on metal content and only 0.05 mag brighter with respect to the recent estimates by Gratton et al. (1997).

One can finally connect theoretical results concerning HB stars with previous predictions about the TO magnitudes to give a theoretical calibration of the difference in magnitude between $\mathrm{HB}$ and $\mathrm{TO}, \Delta V(\mathrm{TO}-\mathrm{HB})$, often used as age indicator for galactic globulars. This is shown in Fig. 9, where we report $\Delta V(\mathrm{TO}-\mathrm{HB})$ as a function of the age for the four selected assumptions about stellar metallicity.

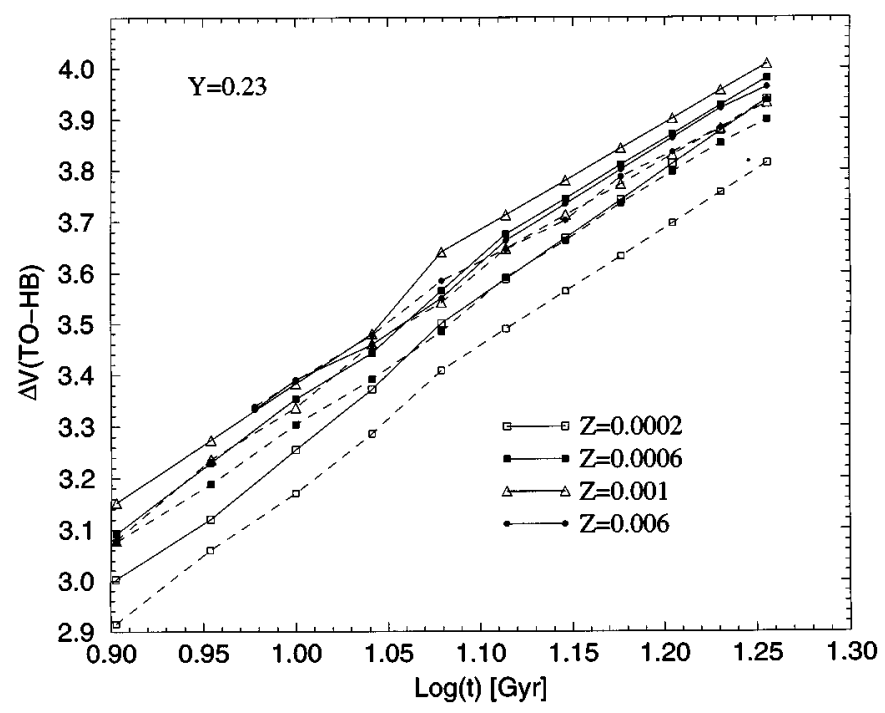

Fig. 9. The difference in magnitude between HB and TO as a function of the age for present models with (solid line) and without (dashed line) element diffusion for the labeled values of metallicities

\section{The role of the original He content}

All the theoretical computations referred in the previous sections assume an amount of original He content equal to $Y=0.23$, which is, at the present, the most popular and widely adopted estimate for the original He abundance in metal poor, Population II stars. However, in Paper I we already discussed the large uncertainty in the evolutionary determination of this parameter. According to such an evidence, we will investigate the role played by the assumption about $Y$ on the present theoretical scenario. To this purpose we reinvestigated the evolutionary behavior of the $Z=0.001$ models (with element diffusion) but under the two alternative assumptions $Y=0.21$ or $Y=0.25$.

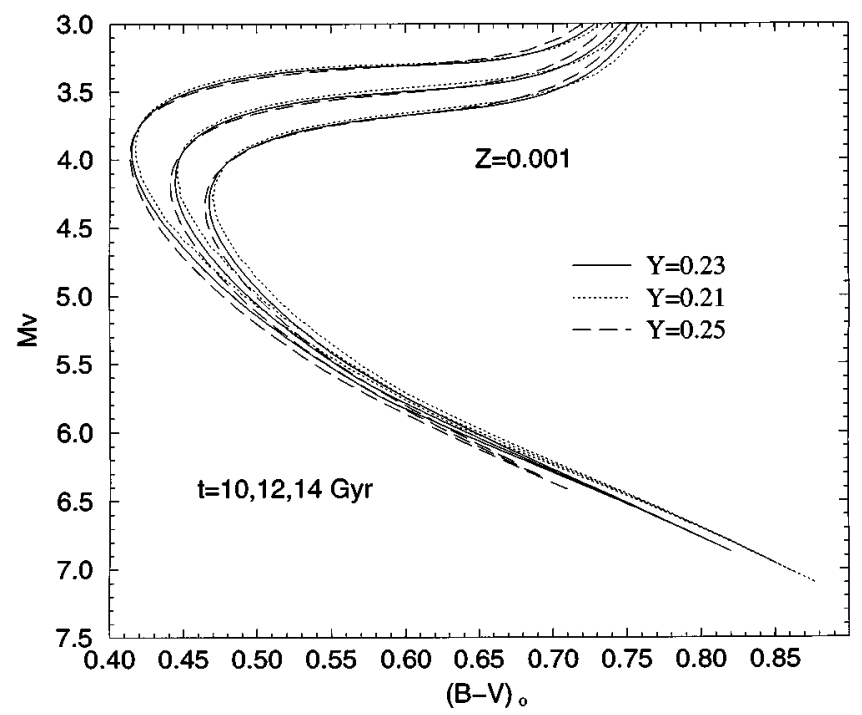

Fig. 10. Present isochrones in the CM diagram for $Z=0.001$, and the labeled values of age and original He abundances

Figure 10 compares selected isochrones for $Z=0.001$ and for the three adopted values of helium abundance. As a result one finds the following relations:

unevolved MS (at $B-V=0.6)$ :

$\Delta M_{v} / \Delta Y \sim 2.5$ (slowly depending on $(B-V)$

TO (at $t=10 \mathrm{Gyr})$ :

$\Delta M_{v}=0.019(Y=0.21 \div 0.23)$

$\Delta M_{v}=0.034(Y=0.23 \div 0.25)$

$\mathrm{HB}\left(\right.$ at $\left.\log T_{\mathrm{e}}=3.85\right)$ :

$\Delta M_{v}=-0.084(Y=0.21 \div 0.23)$

$\Delta M_{v}=-0.068(Y=0.23 \div 0.25)$.

As a whole, present results (where element sedimentation is taken into account) appear in reasonable concordance with canonical evaluations given by Renzini (1991) for the TO magnitudes and by Buzzoni et al. (1983) or, more recently, by Bono et al. (1995) for HB luminosities. One finds that a variation of $Y$ within the assumed limits $(Y=0.23$ $\pm 0.02)$ gives a maximum variation of $0.034 \mathrm{mag}$ in the TO magnitude and of $0.10 \mathrm{mag}$ in the difference of magnitude between TO and HB. According to the calibration given in previous sections this uncertainty implies an error of about 0.2 Gyr for ages from the TO magnitude, and an error of about 1 Gyr for ages from the "vertical" method, i.e., from the difference in magnitude between TO and $\mathrm{HB}$. 
From the previous sections one also derives $\Delta M_{v}(\mathrm{TO}) \sim 0.37 \Delta[\mathrm{Fe} / \mathrm{H}]$ and $\Delta M_{v}=0.18 \Delta[\mathrm{Fe} / \mathrm{H}]$. Assuming an uncertainty of \pm 0.2 dex on current evaluations of cluster metallicity, we obtain that such an error drives, by itself, a variation of, about, $0.08 \mathrm{mag}$ in the predicted TO, and of about $0.04 \mathrm{mag}$ on the difference in magnitude between TO and HB. As a result, one finds that with the assumed uncertainty in both Helium and metal content, even with perfect photometry, the age of a cluster cannot be determined better than \pm about 0.8 Gyr from the TO magnitude or better than, about, 1.4 Gyr from the vertical method.

We remind that in all cases we assumed scaled solar composition as given by Grevesse \& Noels (1993). However, for metallicity not too much high, the effect of the $\alpha$-enhancement on the evolutionary tracks and isochrones can be simulated by using a scaled solar mixture of total metallicity equal to the actual one (see Salaris et al. 1993). Detailed calculations recently performed by Salaris \& Weiss (1998b) show that the $\alpha$-enhancement does not influence at all the $\Delta V(\mathrm{TO}-\mathrm{HB})$ parameter for metallicities up to $Z \approx 0.004$, while for $Z=0.01$ the variation of $\Delta V(\mathrm{TO}-\mathrm{HB})$ is about $0.1 \mathrm{mag}$. Thus the influence of the $\alpha$-enhancement on $\Delta V(\mathrm{TO}-\mathrm{HB})$ is expected to be negligible up to our highest adopted metallicity $(Z=0.006)$. In the case of $\alpha$-enhancement we only expect, for $Z=0.006$, a shift of the color of the RG branch, well within the known uncertainties due to the uncertainty on the efficiency of superadiabatic convection and on the adopted models atmospheres.

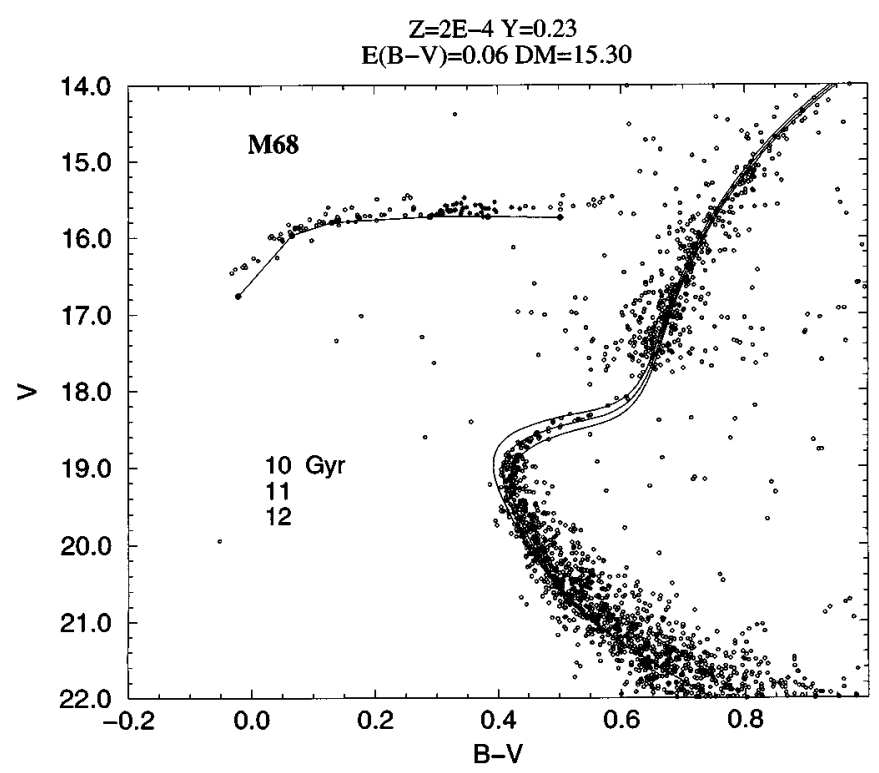

Fig. 11. Isochrones for ages between 10 and 12 Gyr and ZAHB compared to the CMD of M 68 (data from Walker 1994). Composition, distance modulus and reddening used for the fit as labeled. The adopted mixing length is $2.0 \mathrm{Hp}$

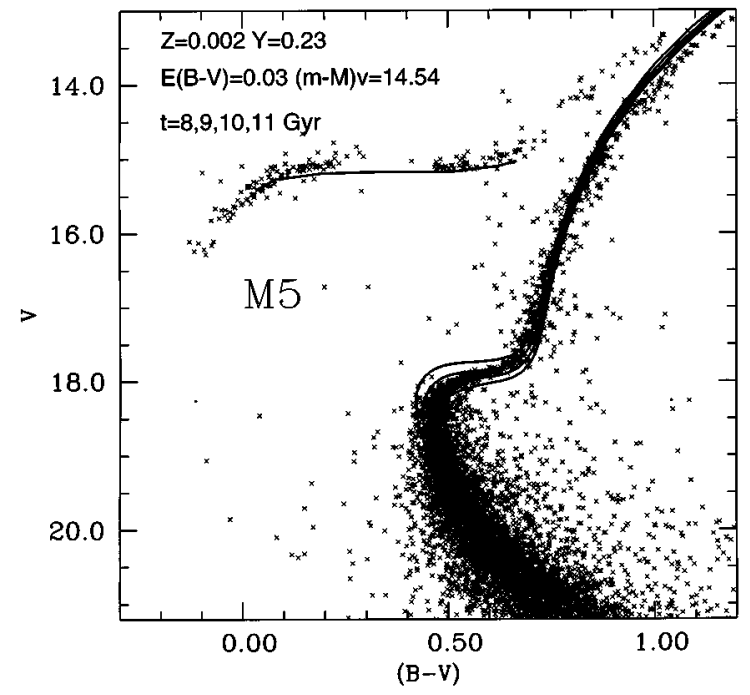

Fig. 12. Isochrones for age between 9 and 11 Gyr and ZAHB compared to the CMD of M 5 (data from Sandquist et al. 1996). Composition, distance modulus and reddening as labeled. The adopted mixing length is $2.3 \mathrm{Hp}$

As already discussed by Caputo et al. (1983) one finally finds that MS and $\mathrm{HB}$ have opposite behavior as far as the amount of original He is concerned. This implies that the difference in magnitude between the HB and the MS at a given color is in principle an indicator of the original He content independent on the cluster age and not affected, as the well known parameter $R$ is, by the still large inaccuracy on the rate of the ${ }^{12} \mathrm{C}-\alpha$ reactions. This indicator is becoming more and more relevant vis-a-vis the increased capability of precise photometry of faint MS stars in galactic globulars. Combining the previous relations one finds $\Delta M_{v}(\mathrm{HB}-\mathrm{MS}) \sim 6.5 \Delta Y$. Thus $\Delta M_{v}(\mathrm{HB}-\mathrm{MS})$ appears rather sensitive to the amount of original He, increasing by $0.07 \mathrm{mag}$ when $Y$ is increased by only 0.01 . However one should remember that significant theoretical uncertainties, due to the uncertainty in the efficiency of superadiabatic convection and to the choice of color transformations, together with observational uncertainties, due to the evaluation of the cluster reddening and metallicity, make de facto this quantity a difficult parameter to be used as helium calibrator.

Table 3. Selected evolutionary quantities (see text) for stellar models with $Y=0.23$ and $Z=0.0006$ without element diffusion. Ages are in Gyr and masses are in solar units

\begin{tabular}{|c|c|c|c|c|c|c|}
\hline$M t^{\mathrm{TO}}$ & $\operatorname{og} L^{\mathrm{TO}}$ & $\operatorname{og} T_{\mathrm{e}}^{\mathrm{TO}}$ & $\operatorname{og} L^{\mathrm{bu}}$ & $t^{\text {flash }}$ & $\operatorname{og} L^{\text {flash }}$ & $M_{\mathrm{c}} \quad Y_{\mathrm{HB}}$ \\
\hline 0.634 .9 & -0.083 & 3.762 & - & 38.56 & 3.375 & 0.50730 .232 \\
\hline 0.719 .4 & 0.107 & 3.785 & 1.956 & 22.40 & 3.389 & 0.50830 .237 \\
\hline 0.811 .5 & 0.278 & 3.808 & 2.019 & 13.85 & 3.384 & 0.50620 .242 \\
\hline 0.97 .4 & 0.460 & 3.834 & 2.091 & 9.12 & 3.365 & 0.50190 .245 \\
\hline 1.05 .1 & 0.638 & 3.870 & 2.133 & 6.33 & 3.372 & 0.50220 .249 \\
\hline
\end{tabular}




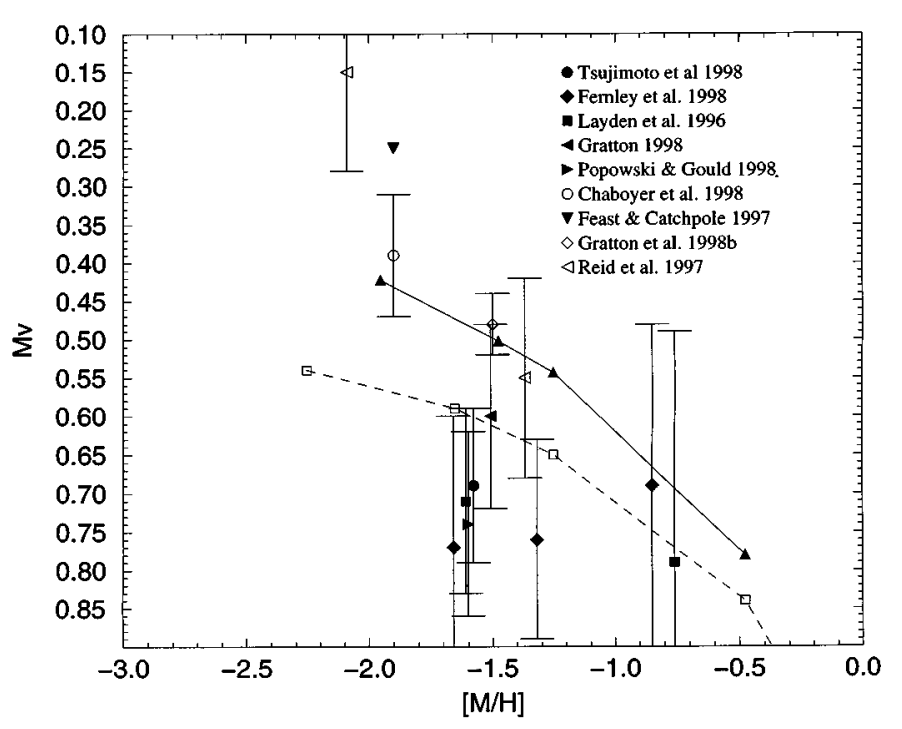

Fig. 13. Theoretical predictions concerning the HB magnitudes from the present paper (full line: diffusion) or from CCP (dashed line) compared with recent observational estimates as derived either from the subdwarf fitting (open symbols) or from RR Lyrae parallaxes (filled symbols)

Table 4. The same as in Table 3 for models with element diffusion

\begin{tabular}{rrrrrrrr}
\hline \hline \\
$M t^{\mathrm{TO}} \log L^{\mathrm{TO}}$ & $\log T_{\mathrm{e}}^{\mathrm{TO}}$ & $\log L^{\text {bump }}$ & $t^{\text {flash }}$ & $\log L^{\text {flash }}$ & $M_{\mathrm{C}}$ & $Y_{\mathrm{HB}}$ \\
\hline & & & & & & & \\
0.631 .9 & -0.137 & 3.752 & - & 36.10 & 3.376 & 0.5192 & 0.200 \\
0.718 .1 & 0.071 & 3.777 & 1.917 & 21.27 & 3.391 & 0.5142 & 0.217 \\
0.810 .8 & 0.251 & 3.802 & 1.990 & 13.36 & 3.388 & 0.5094 & 0.229 \\
0.96 .95 & 0.428 & 3.829 & 2.065 & 8.88 & 3.382 & 0.5063 & 0.237 \\
1.04 .9 & 0.619 & 3.862 & 2.126 & 6.20 & 3.374 & 0.5037 & 0.243 \\
\hline \hline
\end{tabular}

\section{Discussion and final remarks}

Before closing the paper let us use the presented theoretical scenario to fit the $\mathrm{C}-\mathrm{M}$ diagram of selected globular clusters. For a first test we chose $\mathrm{C}-\mathrm{M}$ diagram presented by Walker (1994) for the metal poor cluster M 68 . A preliminary discussion on that matter has been recently presented by Brocato et al. (1997) on the basis of Paper I evolutionary results and using Kurucz (1992) transformations. Without repeating the discussion given in that paper we present in Fig. 11 the best fitting of the observational diagram as obtained on the basis of our $Z=0.0002$ isochrones with element diffusion, adopting the very last version of Kurucz's model atmosphere (Castelli 1997a,b), where the new solar abundances and the enhancement of $\alpha$-elements have been taken into account.

The best fitting is achieved for an age of about 11 Gyr, with distance modulus and reddening as labeled. Note that to fit the color of the RG branch we adopted $l=2.0 \mathrm{Hp}$. However, Fig. 11 shows that with such an
Table 5. Selected TO quantities for $\mathrm{H}$ burning isochrones with $Y=0.23$ and $Z=0.0006$, with and without element diffusion for the labeled values of age (Gyr)

\begin{tabular}{|c|c|c|c|}
\hline$t^{\mathrm{TO}}$ & $\log L^{\mathrm{TO}}$ & $\log T_{\mathrm{e}}^{\mathrm{TO}}$ & $M^{\mathrm{TO}}\left[M_{\odot}\right]$ \\
\hline \multicolumn{4}{|c|}{ standard } \\
\hline 8 & 0.510 & 3.831 & 0.893 \\
\hline 9 & 0.461 & 3.824 & 0.865 \\
\hline 10 & 0.418 & 3.818 & 0.840 \\
\hline 11 & 0.373 & 3.812 & 0.818 \\
\hline 12 & 0.320 & 3.807 & 0.798 \\
\hline 13 & 0.288 & 3.803 & 0.781 \\
\hline 14 & 0.264 & 3.800 & 0.766 \\
\hline 15 & 0.242 & 3.797 & 0.753 \\
\hline 16 & 0.216 & 3.794 & 0.740 \\
\hline 17 & 0.192 & 3.791 & 0.728 \\
\hline 18 & 0.169 & 3.789 & 0.717 \\
\hline \multicolumn{4}{|c|}{ diffusion } \\
\hline 8 & 0.473 & 3.823 & 0.883 \\
\hline 9 & 0.420 & 3.816 & 0.854 \\
\hline 10 & 0.381 & 3.809 & 0.831 \\
\hline 11 & 0.339 & 3.803 & 0.809 \\
\hline 12 & 0.294 & 3.799 & 0.789 \\
\hline 13 & 0.265 & 3.795 & 0.772 \\
\hline 14 & 0.238 & 3.791 & 0.757 \\
\hline 15 & 0.207 & 3.787 & 0.743 \\
\hline 16 & 0.184 & 3.784 & 0.730 \\
\hline 17 & 0.162 & 3.781 & 0.718 \\
\hline 18 & 0.122 & 3.778 & 0.706 \\
\hline
\end{tabular}

assumption the MS appears slight bluer than observed, requiring lower values of the mixing length parameter and indicating that the CM diagram of the cluster can hardly be fitted with precision assuming a constant mixing length for all the evolutionary phases. By neglecting this (false) problem of temperature of cool stars, one finds that the best fitting requires a cluster distance modulus $\mathrm{DM}=15.30$, in excellent agreement with Gratton et al. (1997) who used Hipparcos parallaxes to find for the cluster $\mathrm{DM}=15.31$ and a (mean) age of $11.3 \mathrm{Gyr}$.

As a test of theory at larger metallicity, Fig. 12 present the best fitting of the $\mathrm{C}-\mathrm{M}$ diagram presented by Sandquist et al. (1996) for the intermediate metallicity cluster M 5. Now the best fitting gives an ages of about 10 Gyr and a distance modulus $\mathrm{DM}=14.54$. On the basis of Hipparcos parallaxes Gratton et al. (1997) give DM = $14.60 \pm 0.07$ with a (mean) age of 10.5 Gyr. On the same ground, Chaboyer et al. (1998) gives DM = $14.51 \pm 0.09$ with an age of $8.9 \pm 1.1 \mathrm{Gyr}$. 
Table 6. Selected evolutionary quantities for ZAHB models with $Z=0.0006$

\begin{tabular}{|c|c|c|}
\hline \multicolumn{3}{|c|}{ standard } \\
\hline \multicolumn{3}{|c|}{$Z=0.0006 Y_{\mathrm{HB}}=0.242 M_{\mathrm{c}}=0.5062 M_{\odot}$} \\
\hline$M\left[M_{\odot}\right]$ & $\log L \log T_{\mathrm{e}}$ & $t_{\mathrm{He}}[\mathrm{Myr}]$ \\
\hline 0.53 & $1.347 \quad 4.355$ & 95.8 \\
\hline 0.55 & $1.392 \quad 4.275$ & 91.4 \\
\hline 0.60 & $1.548 \quad 4.127$ & 85.3 \\
\hline 0.65 & 1.6813 .938 & 81.5 \\
\hline 0.70 & $1.743 \quad 3.770$ & 79.3 \\
\hline 0.75 & $1.786 \quad 3.731$ & 78.8 \\
\hline \multicolumn{3}{|c|}{ diffusion } \\
\hline \multicolumn{3}{|c|}{$Z=0.0006 Y_{\mathrm{HB}}=0.229 M_{\mathrm{c}}=0.5094 M_{\odot}$} \\
\hline$M\left[M_{\odot}\right]$ & $\log L \log T_{\mathrm{e}}$ & $t_{\mathrm{He}}[\mathrm{Myr}]$ \\
\hline 0.53 & $1.353 \quad 4.364$ & 94.5 \\
\hline 0.55 & $1.394 \quad 4.276$ & 90.2 \\
\hline 0.60 & $1.526 \quad 4.131$ & 84.4 \\
\hline 0.65 & $1.663 \quad 3.938$ & 80.9 \\
\hline 0.70 & $1.726 \quad 3.775$ & 79.4 \\
\hline 0.75 & $1.762 \quad 3.734$ & 76.8 \\
\hline 0.80 & $1.786 \quad 3.724$ & 76.7 \\
\hline
\end{tabular}

The presented evolutionary models appears in both cases to give excellent agreement with independent evaluations of the cluster distance moduli based on Hipparcos data. This strongly increases the confidence in HB stars as (theoretical) standard candles and, in the same time, in the reliability of the derived cluster ages. One may notice that both clusters we are dealing with have been recently fitted with less updated physics, corresponding to step 4 in Paper I. In that case it was derived $t=12.2$ Gyr for M 68 (Salaris et al. 1997) and, with slight different assumptions about the cluster chemical composition $(Y=0.235$, $Z=0.0015), t=10.9$ Gyr for M 5 (Salaris \& Weiss 1998a). Comparison with present results casts new light on the further rejuvenation of cluster ages induced by both the subsequent updating of the physics and the introduction of the element diffusion.

One can safely assume a conservative error of $\pm 0.1 \mathrm{mag}$ in our estimates of the difference in magnitude between TO and $\mathrm{HB}$, due to the arbitrarity introduced when the theoretical $\mathrm{HB}$ and isochrones are fitted to an observational CM diagram. As an example, we note that Brocato et al. (1997) using the theoretical models of Paper I, find for M 68 a distance modulus $(\mathrm{DM}=15 \pm 0.25)$ slightly different from the present result, due to the slightly different way in which the theoretical $\mathrm{HB}$ is fitted to the observational one. This variation in the distance modulus together with the adopted color transformations (which influence the look of the fit) yields a difference of $\sim 1$ Gyr in the estimated age. With the above quoted assumptions about the uncertainty in the chemical composition we conclude that the adopted theoretical scenario gives for our clusters:

M 68: $t=11 \pm 1.4 \pm 1.0 \mathrm{Gyr}$

M 5: $t=10 \pm 1.4 \pm 1.0 \mathrm{Gyr}$

where the first error is due to the uncertainty in the chemical composition, while the second represents the uncertainty in the fit. In passing, we note that the close similarity between the $\mathrm{C}-\mathrm{M}$ diagrams of M 68 and M 92 (as, e.g., discussed in Brocato et al. 1997 and Salaris et al. 1997) drives to the conclusion that both clusters should have quite similar ages. According to the above discussion we suggest for these very metal poor globulars an age of the order of 11 Gyr against the 14 Gyr recently derived by Pont et al. (1998). One may notice that the above age evaluations could suggest a possible correlation between cluster ages and metallicity, the more metallic cluster being also the younger one. The evidence from the figure appears clear enough, however the source of possible errors in the photometry and in the fitting do not allow firm conclusions about a problem which deserves much more accurate investigations.

As a final remark, let us here remind once more that the above age estimates rely on the theoretically predicted HB luminosity. We have already quoted the good agreement of such a prediction with cluster distance moduli as derived by the fitting of Hipparcos subdwarf magnitudes. However one has also to remind that several estimates of RR Lyrae luminosities based on Hipparcos data give sensitively fainter magnitudes. As shown in Fig. 13, the issue is far from being clearly settled. Here we can only say that if such faint magnitudes will be eventually confirmed, present theory is obviously overestimating the He cores at the end of RG evolution, likely as a consequence of a corresponding overestimate of the efficiency of cooling along the RG phase. In this case, data in Table 1 indicate that cluster ages should be increased by $\Delta \log t \sim 0.4 \delta M_{v}$, where $\delta M_{v}$ represents the difference between the actual and the predicted magnitudes of RR Lyrae stars.

Acknowledgements. It is a pleasure to thank Giuseppe Bono for a critical reading of the manuscript and for valuable suggestions. One of the authors, S.C., acknowledges the grant from C.N.A.A.

\section{Appendix}

Table 3 lists selected evolutionary quantities for the new models with $Z=0.0006$ when the efficiency of element sedimentation is neglected. Left to right one finds: mass of the model, age, luminosity and effective temperature at the track Turn-Off (TO), mean luminosity of the 
RGB "bump", age at the He flash and the corresponding luminosity, mass of the He core and amount of extra-He brought to the surface at this time. Table 4 gives the same quantities but for models where element sedimentation is taken into account according to the procedure reported in Paper I. Table 5 gives details on the isochrone TO over the explored range of ages for the new models with $Z=0.0006$. Table 6 gives selected evolutionary quantities for the new HB models with $Z=0.0006$.

Finally we compare the evolutionary characteristics of our models with those of similar models, for the same chemical composition and slightly different physics, recently presented by Straniero et al. (1997) finding a substantial agreement. Regarding the $\mathrm{H}$ burning phases, there is a good agreement in the luminosity and effective temperature of the TO region, while our TO ages are lower by about $10 \%$, presumably due to the adoption of a different EOS. As far as the evolutionary characteristics at the He flash is concerned, the value of the extrahelium brought to the surface during the first dredge-up is the same, while our He cores are larger of about $0.005 M_{\odot}$. This very slight difference could be due to our adoption of updated calculations for plasma neutrino energy losses (Haft et al. 1994).

\section{References}

Alonso A., Arribas S., Martinez-Roger C., 1996, A\&A 313, 873 Bono G., Castellani V., Degl'Innocenti S., Pulone L., 1995, A\&A 297, 115

Bono G., Caputo F., Castellani V., Marconi M., 1997, A\&AS 121, 327

Brocato E., Castellani V., Piersimoni A., 1997, ApJ 491, 789

Brocato E., Castellani V., Villante F., 1998, MNRAS (in press)

Buser R., Kurucz R.L., 1978, A\&A 70, 555

Buser R., Kurucz R.L., 1992, A\&A 264, 557

Buzzoni A., Fusi Pecci F., Buonanno R., Corsi C.E., 1983, A\&A 128, 94

Caloi V., D'Antona F., Mazzitelli I., 1997, A\&A 320, 823

Canuto F., Mazzitelli I., 1991, ApJ 370, 295

Caputo F., Cayrel R., Cayrel de Strobel G., 1983, A\&A 123, 135

Caputo F., Martinez Roger C., Paez E., 1987, A\&A 183, 228

Carney B.W., Storm J.J., Rodney V., 1992, ApJ 386, 663

Carretta E., Gratton R.G., 1997, A\&AS 121, 95

Cassisi S., Salaris M., 1997, MNRAS 285, 593

Cassisi S., Castellani V., Degl'Innocenti S., Weiss A., 1998, A\&AS 129, 267

Castellani V., Quarta M.L., 1987, A\&AS 71, 1
Castellani V., Chieffi S., Pulone L., 1991, ApJS 76, 911

Castelli F., Gratton R.G., Kurucz R.L., 1997a, A\&A 318, 841

Castelli F., Gratton R.G., Kurucz R.L., 1997b, A\&A 324, 432

Chaboyer B., 1995, ApJ 444, L9

Chaboyer B., Demarque P., Kernan P.J., Krauss L.M., Sarajedini A., 1996, MNRAS 283, 683

Chaboyer B., Demarque P., Kernan P., Krauss L.M., 1998, ApJ 494, 96

Clementini G., Carretta E., Gratton R.G., et al., 1995, AJ 110, 2319

D'Antona F., Caloi V., Mazzitelli I., 1997, ApJ 477, 519

De Santis R., 1996, A\&A 306, 755

Feast M.W., Catchpole R.M., 1997, MNRAS 286, L1

Fernley J., Barnes T.G., Skillen I., et al., 1998, A\&A 330, 515

Gratton R.G., 1998, preprint astro-ph/9710271 to appear on MNRAS

Gratton R.G., Fusi Pecci F. Carretta E., et al., 1997, ApJ 491, 749

Gratton R.G., Carretta E., Castelli F., 1998a, A\&A (in press)

Gratton R.G., Clementini G., Fusi Pecci F., Carretta E., 1998b, in: "Views on distance indicators", Caputo F. (ed.) Mem. SAIt (in press)

Grevesse N., Noels A., 1993 in: "Origin and evolution of the elements", Prantzos N., Vangioni-Flam E., Cassé M. (ed.). Cambridge University Press, Cambridge, p. 15

Haft M., Raffelt G., Weiss A., 1994, ApJ 425, 222

Kurucz R.L., 1992, in IAU Symp. 149, The stellar Population of Galaxies, Barbuy B., Renzini A. (eds.). Dordrecht: Kluwer, p. 225

Layden A.C., Hanson R.B., Hawley S. L., Klemola A.R., Hanley C.J., 1996, AJ 112, 2110

Mazzitelli I., D'Antona F., Caloi V., 1995, A\&A 302, 382

Pont F., Mayor M., Turan C., VandenBerg D.A., 1998, A\&A 329,87

Popowski P., Gould A., preprint astro-ph/9802168

Reid I.N., 1997, AJ 114, 161

Renzini A., 1991, in: "Observational tests of cosmological inflation", Shanks T. et al. (ed.). Dordrecht: Kluwer, p. 7

Salaris M., Weiss A., 1998a, A\&A (in press)

Salaris M., Weiss A., 1998b, A\&A (submitted)

Salaris M., Chieffi S., Straniero O., 1993, ApJ 414, 580

Salaris M., Degl'Innocenti S., Weiss A., 1997, ApJ 479, 665

Sandage A., 1993, ApJ 106, 703

Sandquist E.L., Bolte M., Stetson P.B., Hesser J.E., 1996, ApJ 470,910

Straniero O., Chieffi A., Limongi M., 1997, ApJ 490, 425

Tsujimoto T., Miyamoto M., Yoshii Y., 1998, ApJ 492, L79

VandenBerg D.A., Bolte M., Stetson P.B., 1996, ARA\&A 34, 461

Walker A.R., 1992, ApJ 390, L81

Walker A.R., 1994, AJ 108, 555 